\title{
Interpreting the dynamic equilibrium during evaporation in a Caesium environment
}

\author{
M. Fadone ${ }^{1, a)}$, M. Barbisan ${ }^{1}$, S. Cristofaro², M. De Muri ${ }^{1}$, G. Serianni ${ }^{1}$, E. Sartori ${ }^{1}$ \\ ${ }^{1}$ Consorzio RFX, Associazione EURATOM-ENEA sulla fusione, c.so Stati Uniti 4, 35127, Padova, Italy \\ ${ }^{2}$ Max-Planck-Institut für Plasmaphysik, Boltzmannstr. 2, 85748 Garching, Germany
}

(Presented XXXXX; received XXXXX; accepted XXXXX; published online XXXXX)

(Dates appearing here are provided by the Editorial Office)

The Caesium ovens for the prototype source of the ITER neutral beam injectors are currently tested in the CATS facility, with a background pressure of $10^{-6} \mathrm{mbar}$. Different diagnostics are here installed: two Langmuir-Taylor detectors allow to determine the Cs vapour evaporation rate from the oven and the Cs density at different positions in the vacuum chamber; laser absorption spectroscopy is used to measure the density integrated over a line of sight and a quartz crystal microbalance to detect the cesium mass deposited in time over a surface. In this paper, we present a model to describe the dynamic equilibrium in the evaporation chamber of CATS during Cs evaporation with the first oven tested in order to gain information about the Cs sticking coefficient at the walls. The model hence includes sticking and energy accommodation of the Cs atoms to the walls, calculates the flux density at the surfaces and provides the Cs atom density at any location in the volume. By this model, we simulate the Cs evaporation and the equilibrium density, comparing the modeled results with the experimental data. As a result, a sticking coefficient of $2 \%$ is obtained.

\section{INTRODUCTION}

ITER neutral beam injectors (NBI) must deliver a total power of $33 \mathrm{MW}$ into the fusion plasma [1]. The NBI consists of an RF hydrogen (or deuterium) plasma source, an extraction region which extracts negative ions, followed by an acceleration and neutralization stage. The negative ions extracted from the plasma source must be accelerated up to $1 \mathrm{MeV}$, with an extracted deuterium current density of $286 \mathrm{~A} / \mathrm{m}^{2}$ [1]. Negative ion sources for fusion are based on the surface formation of negative hydrogen ions by impact of atomic hydrogen or positive hydrogen ions onto a low work function surface [2]. To lower the surface work function and enhance the formation of negative ions, Caesium is evaporated inside the source [3] thanks to its low work function $(2.1 \mathrm{eV})$ and its particular disposition configuration on a surface [4]). Cs is evaporated inside the ion source by means of Cs oven, whose nozzle determines the Cs distribution during the vacuum phase. The Cs deposition on the source surfaces is however affected by the cleanliness quality and temperatures of the walls [5]. Additionally, when there is interaction between the plasma and the surfaces, Cs is subjected to a redistribution and removal from the surfaces [6]. Finally, a potential issue is where the deposition of Cs takes place: if part of the evaporated Cs goes beyond the designed plasma grid of the negative ion source, the voltage holding between the unwanted caesiated surfaces and the rest of the system is not guaranteed anymore. SPIDER (Source for Production of Ions of Deuterium Extracted from Radio frequency plasma) is the ITER plasma source prototype with the purpose to test and optimize a $110 \mathrm{kV}$ negative ion beam source [7] and is located at the Neutral Beam Test Facility (NBTF) at the Consorzio RFX site in Padova, Italy. Three Cs ovens will be installed at the back-plate of the plasma source [8] and have been recently manufactured. First tests of Cs evaporation are performed at the CATS test facility and a model is applied to gain information about the sticking probability. This last parameter is the probability of a

a) Author to whom correspondence should be addressed:

michele.fadone@igi.cnr.it particle hitting a surface to stay attached to it. Finding the correct sticking coefficient to simulate the actual Cs distribution in a vacuum environment is of high importance for ITER NBI experiments. Therefore, the final aim of this paper is to find the empirical sticking coefficient through numerical simulations that best match the experimental data collected through a Cs evaporation facility located always in Padua. This paper is a first step to deal with the first Cs evaporation without plasma in a vacuum chamber in order to foresee what can happen in SPIDER experiment for ITER.

\section{CAESIUM TEST STAND}

CAesium Test Stand (CATS) is the facility located at Consorzio RFX to test the reliability of the three Cs ovens for SPIDER and to characterize the Cs evaporation [8]. As visible in Figure 1, the facility consists of a stainless steel vacuum vessel with $400 \mathrm{~mm}$ of

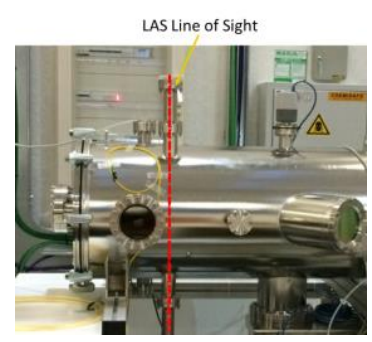

(a)

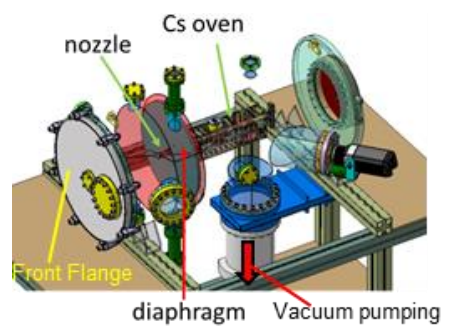

(b)
Figure 1. a) CATS vacuum chamber with the line of sight of the LAS. b) Inside view of CATS vacuum vessel, with the diaphragm and vacuum pumping highlighted.

diameter and $1070 \mathrm{~mm}$ in length. A diaphragm is located at $300 \mathrm{~mm}$ from the front flange and separates the vacuum vessel in a evaporation region (front part where there is the nozzle) and a rear part where the vacuum pump and the oven are hosted. The diaphragm, which is centrally located on the symmetry axis of 


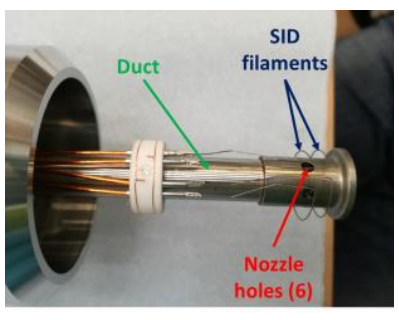

(a)

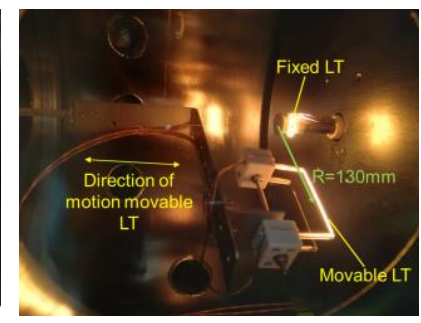

(b)
Figure 2. a) Cesium oven prototype nozzle. The 6 holes nozzle through which Cs evaporates and the duct are highlighted. b) Fixed and movable LT detectors during CATS operation

CATS, has not the same diameter of the vacuum vessel, but its radius is $20 \mathrm{~mm}$ shorter. The pressure guaranteed during operation is $10^{-6} \mathrm{mbar}$ by means of a $4001 / \mathrm{s}$ turbomolecular pump coupled to a $17 \mathrm{~m}^{3} / \mathrm{h}$ scroll pump. The location of the system is highlighted in Figure $1 \mathrm{~b}$. The surface temperature of the diaphragm is assumed to be $350 \mathrm{~K}$. The temperature of the duct can be raised up to $573 \mathrm{~K}$, but for the later simulations is considered $473 \mathrm{~K}$. The other surfaces of the evaporation region are assumed to be $320 \mathrm{~K}$.

The diagnostics used are (i) Langmuir Taylor (LT) detectors, one fixed around the oven nozzle (see Figure 2a) and one movable along the longitudinal axis of the vessel; (ii) a Laser Absorption Spectroscopy (LAS) and (iii) a Quartz Crystal Microbalance (QCM) and they are described in the following sections.

\section{A. LT detectors}

LT detectors consist of two tungsten filaments of $0.3 \mathrm{~mm}$ diameter. When they are in vacuum, a current power supply guarantees enough current to make them incandescent, i.e. 3.2A for a filament length around $5 \mathrm{~cm}$ means the temperature filament is greater than 1000K [9]. When Cs hit the first filament, it can be ionized [10]. The ionization probability depends on the coverage state of the filament: at the temperature indicated previously, the filament can be considered clean, therefore the ionization probability is $100 \%$ [10]. It is possible then to collect a current of $\mathrm{Cs}^{+}$ions polarizing positively the filaments. From the current it is possible to find the Cs flux and/or the Cs density:

$$
\begin{gathered}
\Gamma=\frac{I}{A q} \\
n=\frac{4 \Gamma}{\bar{v}} \\
\dot{m}=\frac{I}{q} m_{C s}
\end{gathered}
$$

Eq. 1 gives the specific flux $\Gamma$, expressed in $\frac{1}{\mathrm{~m}^{2} \mathrm{~s}}$, from the current $I$ detected, with $A$ the collecting lateral surface of the filament and $q$ the elementary charge. Through Eq. 2 it is possible to find the local Cs density $n$, assuming a temperature $\mathrm{T}=320 \mathrm{~K}$ for the particle thermal velocity $\overline{\mathrm{v}}$. These first 2 equations refer to the current detected by the movable LT detector. As visible in Figure $2 b$, the movable filament is located near the external walls at a mean radius of $130 \mathrm{~mm}$. Moreover, its axial position can be varied, but during CATS operation it has been left always $100 \mathrm{~mm}$ from the diaphragm. So it is safe to assume that temperature of Cs particles detected is similar to the external surfaces one. Finally, for the fixed LT measurements, Eq. 3 shows the relation of the mass flow rate $\dot{\mathrm{m}}$ with the current $I$, knowing the mass of a $\mathrm{Cs}$ atom $\mathrm{mcs}_{\mathrm{C}}$. For the fixed LT, the current detected must be corrected by a view factor since not all the Cs atoms flowing from the nozzle holes hit the filament: according to [11], this correction factor is $5.4 \%$.

\section{B. LAS measurements}

A tunable diode LAS system is installed to measure the line of sight integrated Cs density. The applied system is described in detail in [12]. The line of sight crosses the volume in vicinity of the nozzle, at $9 \mathrm{~cm}$ from the oven axis as shown in Figure 1. The Cs density is calculated considering an absorption length of $48 \mathrm{~cm}$, which is the LAS Line of Sight (LOS) that sees directly the nozzle. Technically the whole LOS length is $80 \mathrm{~cm}$ because additional tubes with windows have been installed to avoid that Cs could reach the optical components. The diameter of the LAS LOS is $6.5 \mathrm{~mm}$

\section{Quartz Crystal Microbalance}

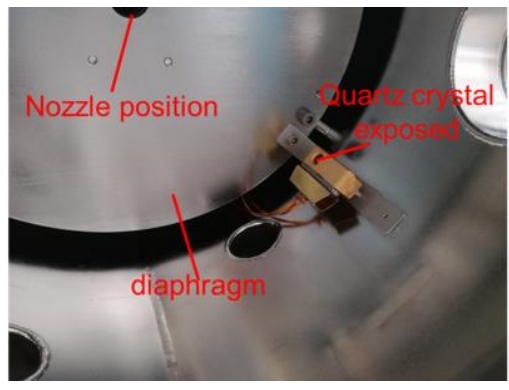

Figure 3. Position of the QCM diagnostic inside CATS vacuum vessel

The diagnostic has been installed in the part of the vacuum chamber where the evaporation occurs, specifically at the lateral wall of the chamber and near the diaphragm. The diagnostic consists of two vibrating quartz crystal electrodes, one exposed to the Cs evaporation while the other not since it is used as reference. The deposition of mass over the first crystal changes its resonance frequency. Thus, measuring the frequency difference in time $\Delta v$, it is possible to find the deposited mass $\Delta m$ and, consequently, an index of sticking:

$$
\begin{gathered}
\Delta m=-\Delta v \frac{A\left(\rho_{q} \mu_{q}\right)^{1 / 2}}{2 v_{0}^{2}} \\
\Gamma_{Q C M}=\frac{\Delta m}{\Delta t} \frac{1}{m_{C s}} \frac{1}{A}
\end{gathered}
$$

where $v_{0}$ is the nominal resonant frequency of the quartz crystal, A is the surface of the electrode, $\rho_{q}$ and $\mu_{q}$ are the density and shear modulus of the crystal and $\Delta t$ is the time exposure considered [13]. The specific flux $\Gamma_{Q C M}$ is used combined to the specific flux recorded through the LT detectors in order to determine the sticking coefficient of the wall in an alternative way.

\section{MODEL DESCRIPTION}

The aim of the paper is to correlate numerical simulation coefficients to experimental data acquired through different diagnostics. The sticking coefficient is necessary to understand the Cs coverage distribution on the surfaces and its density profiles in the vessel. 


\section{A. AVOCADO model}

AVOCADO is a code that allows to study the pressure distribution of a gas [14]. It takes into account only the gas particles-wall collisions with a cosine angle diffusion approach. The code receives a post processed mesh geometry generated by ANSYS software [15] in which the surfaces are assigned to a specific label: in this way it is possible to specify the pressure, the specific pumping speed or the specific gas pumping power on that area. The results of the code are valid only if the Knudsen number $K_{n}=$ $\frac{\lambda}{L}$ (with $\lambda$ the mean free path of the particle and $L$ the characteristic length of the system) is greater than one. Since $\mathrm{L}=300 \mathrm{~mm}$ and $\lambda \approx$ $30 \mathrm{~m}$ for Cs case with a vacuum experimental pressure of $10^{-4} \mathrm{~Pa}$, we have $K_{\mathrm{n}} \gg>1$. The sticking coefficient is introduced by applyingto the surface a negative pumping speed which corresponds to an adsorption rate flux [16]. To determine this negative pumping speed, we have to consider the following relations:

$$
\begin{gathered}
\varphi=\Gamma A=\frac{p A}{\sqrt{2 \pi k_{B} T m_{C S}}}[14] \\
Q=\varphi k_{B} T \\
Q=p S
\end{gathered}
$$

Eq.6 is the evaporation flux contribution $\varphi$ from a wall with temperature $T$, surface $A$, chamber equilibrium pressure $p$ and Boltzmann constant $k_{b}$. Eq. 7 and Eq. 8 show two definitions of the pumping power $Q$, where $S$ is the pumping speed expressed in $\frac{\mathrm{m}^{3}}{\mathrm{~s}}$. Combining Eq. 6, 7 and 8, we obtain the relation for the negative specific pumping speed $\hat{S}[20]$ :

$$
\hat{S}\left[\frac{m}{S}\right]=\frac{S}{A}=\frac{1}{4} \sqrt{\frac{8 k_{B} T}{\pi m_{C S}}} \cdot s=\frac{1}{4} \bar{v} \cdot s
$$

The sticking coefficient $s$ is assumed constant and the temperature dependence is considered in the thermal velocity $\bar{v}$ according to the surface temperatures considered in section II. In closing, the code inputs are the specific flow rate of Cs from the nozzle, the specific pumping speed caused by the real pumping system, the temperatures of the gas and of the various surfaces, which as first approximation are considered equal, as well as their sticking coefficients, the last ones always in terms of negative specific pumping speed.

\section{B. Model of the CAesium Test Stand}

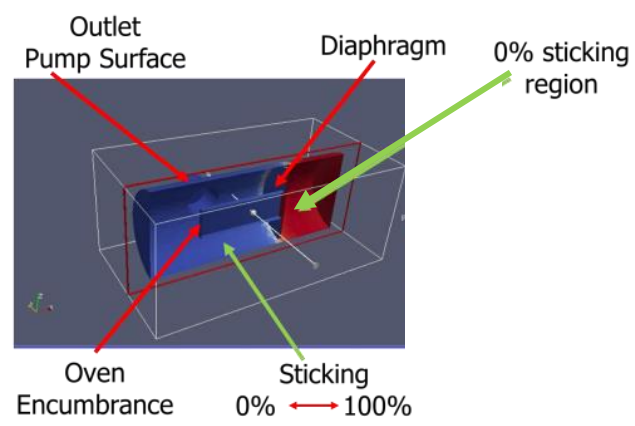

Figure 4. Simple scheme of CATS vacuum vessel. In blue the rear part. In red the front region.

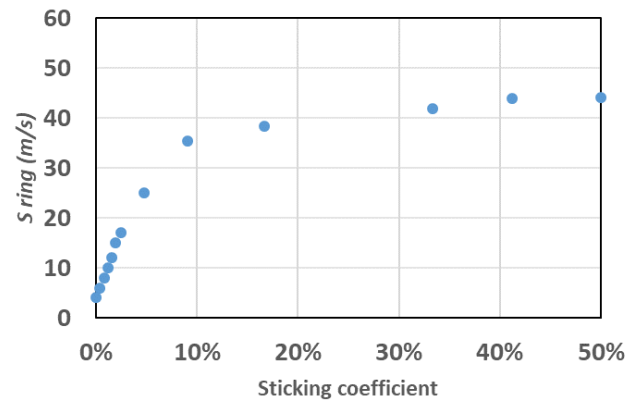

Figure 5. Equivalent specific pumping speed study Sring to apply to the ring gap of the diaphragm for the final 3D model of the evaporation region. $\mathrm{X}$-axis represents the sticking coefficient s.

As written in section II, CATS consists of two volumes in vacuum.At first, the whole vacuum vessel with the oven and diaphragm are considered, as shown in Figure 4. In the rear region the sticking coefficient was varied while in the front part the sticking coefficient was set to $0 \%$. Once the Cs distribution inside the volume is modeled, it is possible to determine the pressure profiles along custom lines inside the volume, in order to compare the model results to the experimental data. Reading from Avocado the pressure and using Eq.8, it was possible to study the effect of the pumping system in the front region (see Figure 5) with the aim to create a final simpler model and reduce the computational time: a vacuum chamber with only the front part and with a pumping speed applied on the ring-shape gap reproducing the effect of the rear part (Figure 6). The front part inputs for Avocado code are the specific Cs mass flow rate from the nozzle, which is put in terms of Q using Eq. 6-7, and the sticking coefficient on the surfaces: 0\% in the nozzle, because during operation its temperature is always over $200^{\circ} \mathrm{C}$ and its inner surfaces can be considered clean, and sticking different for several cases in the rest of the chamber surfaces.

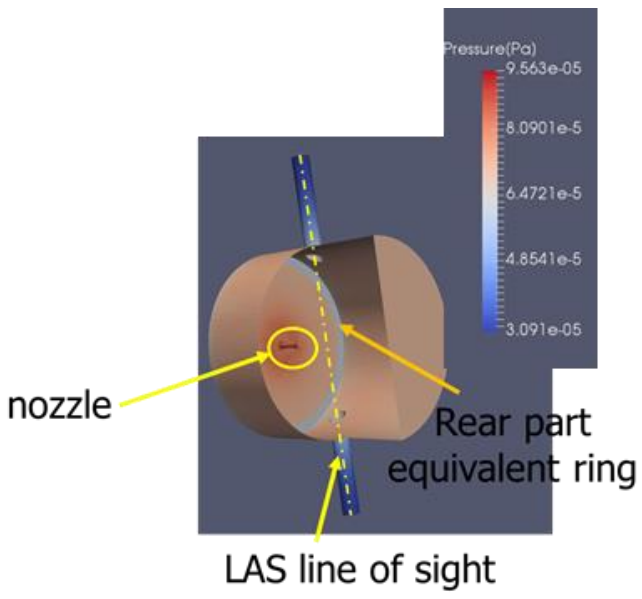

Figure 6. Final configuration 3D model of CATS evaporation region. Plot of the Cs pressure distribution on the surfaces.

\section{RESULTS}

The first result from the numerical simulations is the modeled Cs density profile along the line of view of the LAS for several sticking coefficients (see 


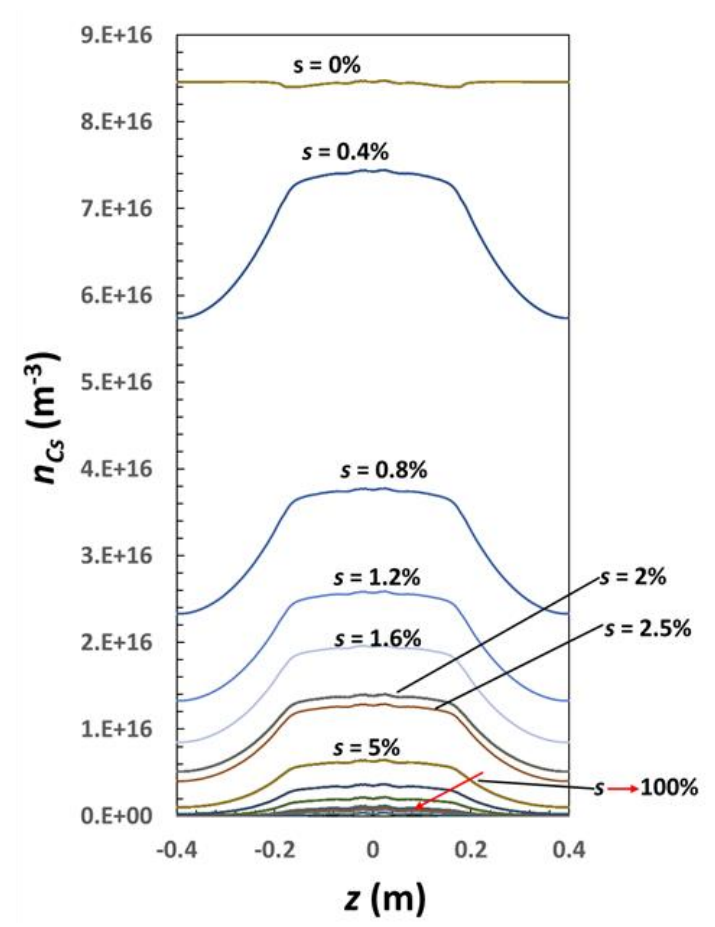

Figure 7. LAS numerical Cs density output (y-axis). x-axis represents the LAS LOS length. The several curves are

obtained varying the sticking coefficient of the surface walls.

Figure 7). This plot is fundamental to analyze the data acquired through the LAS diagnostic. A step of $2 \mathrm{~mm}$ for the line of sight of the LAS has been used so that the integral of the density along the line can be reduced to a simple average of the values found through the simulations. The results show an expected behavior because there is a straight line for $s=0 \%$ while there is a decrease and a change of form of the signal as the sticking increase: the central part of the plot corresponds to the signal from the whole diameter of the vacuum vessel whilst the outer parts of the plot identify the area inside the tubes in between the two windows In addition, over a sticking coefficient of $2 \%$, it is possible to see how the peak density is at least two times the value recorded in the tubes. Finally, the red solid line of Figure 9 represents the densities found through Avocado in the position of the real movable LT detector in CATS: the data have been normalized by the Cs mass flow rate used for the numerical simulation. Figure 8 shows an update of the QCM results obtained in [13]. Knowing that each pure Cs layer consists of $5.2 \cdot 10^{18} \frac{\text { atoms }}{m^{2}}$ [5] and recalling Eq.4-5, it is possible to determine a Cs specific flux to the surface. The ratio between this flux and the one detected by the fixed LT gives an index of th e sticking coefficient allowing the integration of the black dots in Figure 9 . Even if there are only 4 points available, it seems that they follow the trend set by the solid lines (numerical data) with a small offset difference.

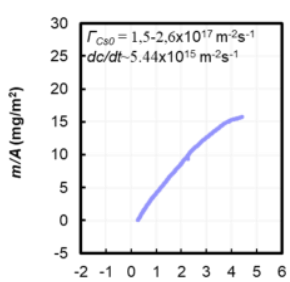

t-t $t_{0}(\mathrm{~h})$

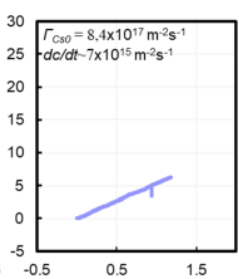

$t-t_{0}$ (h)

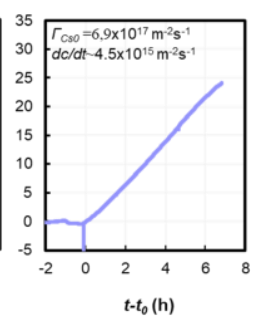

$t-t_{0}(\mathrm{~h})$
Figure 8. QCM post-processing plots for 3 different evaporation experimental sessions

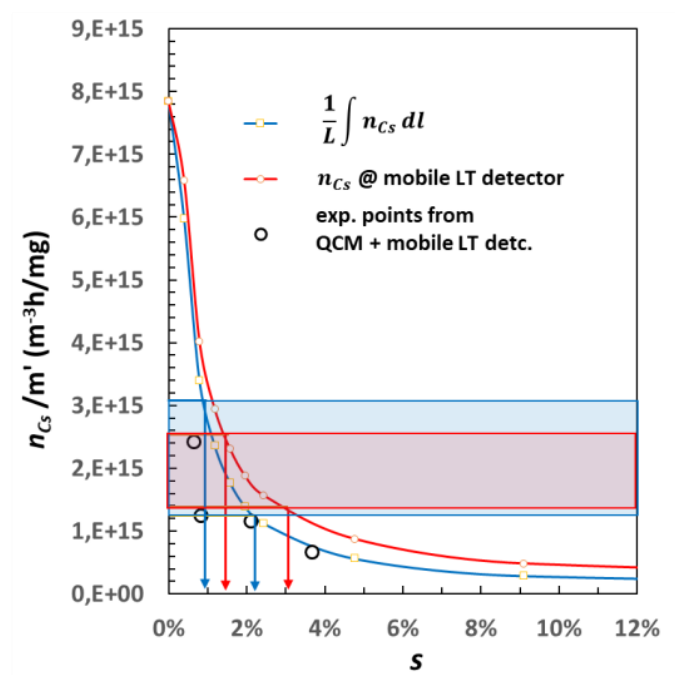

Figure 9. Normalized Cs density by evaporation Cs rate (yaxis) over sticking coefficient $\mathrm{s}$ (x-axis). Numerical data (solid line curves) comparison with experimental data (black dots and rectangle ranges of values found in CATS for different evaporation rates, blue for LAS and red for movable LT)

Figure 9 shows the normalized Cs density in relation to the sticking coefficient. The solid lines represent the numerical simulation results, while the rectangles show the range of values measured in CATS. From both colors, blue for the LAS and red for the movable

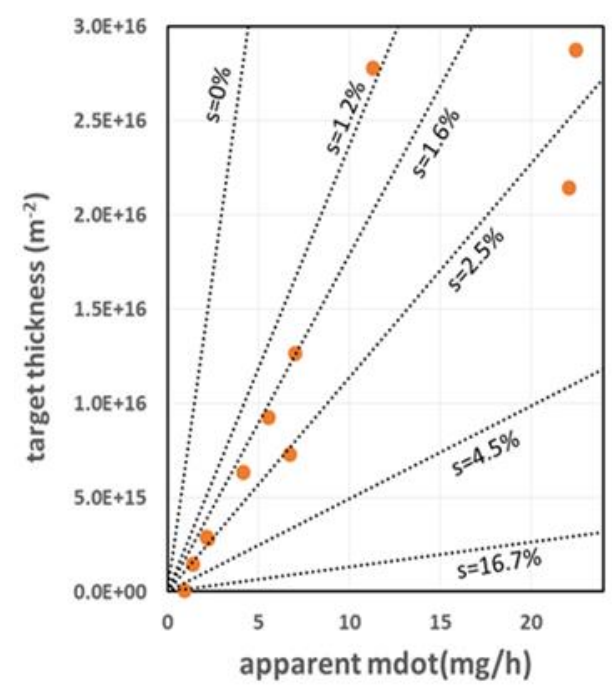

(a)

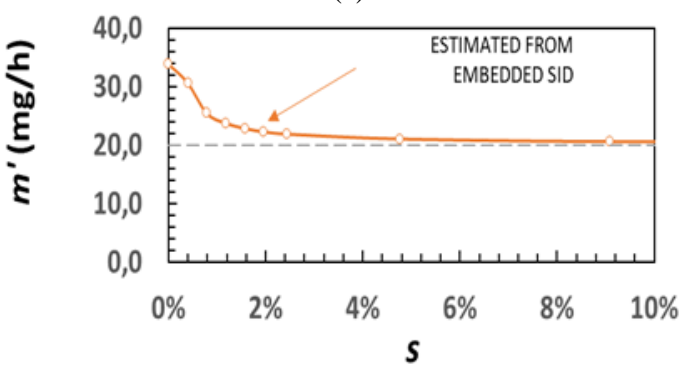

(b)

Figure 10.(a) Target thickness in $\mathrm{m}^{-2}$ (y-axis) comparison of LAS experimental data (orange dots) and numerical data (dashed lines) calculated for different sticking coefficients ( $\mathrm{x}$ axis). (b) Apparent Cs mass flow rate m' (y-axis) according to the sticking coefficient $\mathrm{s}$ (x-axis) 
LT, we see that we always find a sticking coefficient of between $1 \%$ and $3 \%$. Figure 10a shows another approach to compare experimental data with numerical results. At x-axis there is the apparent Cs mass flow rate: it corresponds to the nominal one used for numerical simulations (i.e. $20 \mathrm{mg} / \mathrm{h}$ ) for high sticking coefficients, while it becomes higher when the sticking coefficient decreases (see Figure 10b). This mass flow rate behavior has been obtained through AVOCADO reading the density output over a ring-shape line located in the model right where the fixed LT is actually present on CATS. At the y-axis there is the target thickness, that is, the Cs density multiplied by the length of LAS line of view. Orange dots are the experimental data for several real mass flow rate scenarios corrected with the actual length of $80 \mathrm{~cm}$ (see Figure 7), while the dot lines mark the separation regions of the sticking coefficients, lines that are a result of a combination of Figure $10 \mathrm{~b}$ and the LAS numerical results. As written previously, we can see that the orange dots are focused in the region of $\sim 2 \%$.

\section{CONCLUSIONS}

A facility to test the Cs ovens, which are going to be installed on SPIDER, is operating at the NBTF in Padua. Three type of diagnostics have been used during $\mathrm{Cs}$ evaporation operations to check the reliability of the ovens and to determine the Cs flux and density in the vacuum chamber of the experiment. Meanwhile, by means of Avocado numerical code, several simulations of the facility have been run to match its results with the ones obtained through the diagnostics. The parameter varied in the simulations is the sticking coefficient of the walls of the vacuum chamber. From the comparison between the experimental and numerical results, a sticking coefficient of $2 \%$ has been found. This parameter is going to be used to simulate the SPIDER case in order to foresee the amount of Cs that can pass beyond the plasma grid and deposit on the extraction and/or grounded grid, amount that can generate potential issues.

\section{ACKNOWLEDGMENTS}

The work leading to this publication has been funded partially by Fusion for Energy. This publication reflects the views only of the authors, and F4E cannot be held responsible for any use which may be made of the information contained therein. The views and opinions expressed herein do not necessarily reflect those of the ITER Organization.

Roberto Rizzieri e Diego Ravarotto for problem solving and acquisition system support.

\section{CONTRIBUTORSHIP STATEMENT}

M. Fadone and E. Sartori ran the numerical simulations. G. Serianni and E. Sartori conceived the presented idea for the paper. M. Barbisan, S. Cristofaro, M. de Muri, M. Fadone and E. Sartori followed CATS experimental campaigns. All authors discussed the results and contributed to the final manuscript.

\section{REFERENCES}

${ }^{1} \mathrm{R}$. Hemsworth, et al., Status of the ITER heating neutral beam system, Nucl. Fusion, 49 (2009), p. 045006

${ }^{2} \mathrm{M}$. Bacal and M. Wada, Negative hydrogen ion production mechanisms, Applied Physics Reviews, 2 (2015)

${ }^{3} \mathrm{~V}$. Dudnikov, Forty years of surface plasma source development, Rev. Sci. Instrum., vol. 83, p. 02A708, 2012

${ }^{4}$ E. P. Gyftopoulos and J. D. Levine, Work Function Variation of Metals Coated by Metallic Films, Journal of Applied Physics, 33 (1962)
${ }^{5}$ A. G. Fedorus, A. G. Naumovets, Cesium on Tungsten (011) face; Structure and Work Function, Surface Science, 21 (1970)

${ }^{6}$ E. Sartori, S. Gorno, M. Fadone, and G. Serianni, Analytical study of caesium-wall interaction parameters within a hydrogen plasma, AIP Conference Proceedings 2052, 020006 (2018)

${ }^{7}$ V. Toigo, et al., Nucl. Fusion 57, 8 (2017)

${ }^{8}$ A. Rizzolo, M. Pavei, N. Pomaro, Caesium oven design and R\&D for the SPIDER beam source, Fusion Engineering and Design, 88 (2013)

${ }^{9}$ H. A. Jones and I. Langmuir, G. E. Rev. 30, 354 (1927)

${ }^{10} \mathrm{~J}$. B. Taylor and I. Langmuir, The Evaporation of Atoms, Ions and Electrons from Caesium Films on Tungsten, The Physical Review, 44 (1933)

${ }^{11}$ RFX Internal Technical Note, RFX_SPIDER_TN_450, Modeling the cesium flow from a dispenser oven and the measurement by a surface ionization detector

${ }^{12}$ M. Barbisan, R. Pasqualotto, A. Rizzolo, Design and preliminary operation of a laser absorption diagnostic for the SPIDER RF source, Fusion Engineering and Design (2019, (article in press)

${ }^{13}$ N. Bujanos. Circuit Cellar 91, 66 (1998).

${ }^{14} \mathrm{E}$. Sartori and P. Veltri, AVOCADO: A numerical code to calculate gas pressure distribution, Vacuum 90 (2013), 80-88

${ }^{15}$ Gabriel J. DeSalvo and John A. Swanson. ANSYS Engineering Analysis System User's Manual. Houston, Pa. :Swanson Analysis Systems, 1985.

${ }^{16} \mathrm{~K}$. Jousten, Handbook of Vacuum Technology, Weinheim, Germany, Wiley-VCH Verlag GmbH \& Co. KGaA, 2016(2 ${ }^{\text {nd }}$ edition $)$, p.235 